\title{
Electrocardiografía en equinos fina sangre de carrera
}

\author{
CRISTOBAL DÖRNER S.M. ${ }^{1}$ y ADOLFO GODOY P. ${ }^{1}$
}

1 Hospital Clínico Veterinario de Equinos. Facultad de Ciencias Veterinarias y Pecuarias. Universidad de Chile.

\begin{abstract}
ELECTROCARDIOGRAPHY IN THOROUGHBRED HORSES

The Thoroughbreds horses are highly competitive athletes under great physical demands. To achieve a good performance they need a constant adaptation process of the cardiovascular system to exercise. Within the routine examination of this system, a substantial number of cardiovascular abnormalities can be detected (arrhythmias, murmurs, splitting of heart sounds, etc.), since these animals are competing successfully, it is logical to think that these correspond to the expression of the adaptation process of the cardiovascular system to exercise and not necessarily organically based pathology. Therefore, a very important and useful tool is the collection and interpretation of an electrocardiogram (recording of electrical activity of the heart). Age, training, competition, gender, among others, are variables that affect some of the electrocardiographic parameters. To complete a proper interpretation of the electrical activity generated by cardiac mass, it is necessary to obtain a vector analysis of the electrocardiogram.

Key words: Electrocardiography, Thoroughbred, Exercise, Cardiovascular System.
\end{abstract}

\section{RESUMEN}

Los Equinos Fina Sangre de Carrera son caballos de alta competencia que se encuentran sometidos a grandes exigencias físicas. Para que estos ejemplares obtengan buenos rendimientos es necesario que en ellos ocurra un proceso constante de adaptación al ejercicio. Un número considerable de anormalidades cardiovasculares (arritmias, soplos, desdoblamientos de tonos cardíacos, etc.), pueden ser detectadas al realizar un examen cardiovascular de rutina. Sin embargo, muchos de los ejemplares a los cuales se les detectan dichas alteraciones, se encuentran compitiendo con éxito, por lo que resulta lógico pensar, que estas anomalías corresponden a la expresión del proceso de adaptación cardiovascular al ejercicio y no necesariamente a una patología de base orgánica. Por lo tanto, una herramienta muy importante es el registro e interpretación de un electrocardiograma (registro de la actividad eléctrica del corazón). La edad, entrenamiento, competencia, género, entre otras, son variables que pueden afectar los parámetros electrocardiográficos. Para completar el estudio de la actividad eléctrica generada por la masa cardíaca, es necesario realizar un análisis vectorial a partir del electrocardiograma.

Palabras clave: Electrocardiografía, Fina Sangre de Carrera, Ejercicio, Sistema Cardiovascular.

Universidad de Chile. Casilla 2 Correo 15 La Granja. Santiago, Chile.

E-mail: agodoy@uchile 


\section{INTRODUCCIÓN}

Los equinos Fina Sangre de Carrera son atletas de alta competencia sometidos a grandes exigencias físicas. Para que estos ejemplares logren buenos rendimientos, es necesario que ocurra un proceso de adaptación progresiva de su sistema cardiovascular al ejercicio. Hoy en día, una parte importante del examen físico, dice relación con una evaluación en forma integral de este sistema.

Dentro de la evaluación de rutina del sistema, cardiovascular es normal detectar anomalías a la auscultación y al examen electrocardiográfico (arritmias, soplos, desdoblamiento de tonos cardíacos, entre otros), dado que se trata de animales en competencia incluso con éxito frente a ejemplares que no presentan dichas alteraciones, resulta lógico pensar que estas corresponden a la expresión del proceso de adaptación del sistema cardiovascular al ejercicio y no necesariamente a una patología con base orgánica. Por lo tanto, una parte muy importante y útil es la obtención e interpretación de un registro electrocardiográfico (registro de la actividad eléctrica del corazón), ya que ésta herramienta permite el diagnóstico e interpretación de arritmias, determinación de la frecuencia y tiempo de conducción cardiaco. (Godoy, 2008).

En normalidad, existe un sinnúmero de variables que influyen sobre las características electrocardiográficas, como por ejemplo, edad, entrenamiento, raza, entre otros Para completar una adecuada interpretación de la actividad eléctrica generada por la masa cardíaca, se debe realizar un análisis vectorial del registro electrocardiográfico.

Con este análisis, es posible detectar cambios en la masa miocárdica, en las cámaras cardiacas y desplazamientos cardíacos mediante el análisis de las derivaciones obtenidas en el electrocardiograma (ECG), lo que es de utilidad en el hombre, perro y otras especies, pero sin embargo, el uso de la vectocardiografía en la evaluación clínica de la función cardiaca en el equino es aún discutido.

Un aspecto importante de considerar en el ámbito de los caballos deportistas es lograr dilucidar la o las causas por las cuales se presentan malos rendimientos en los ejemplares, situación que hoy en día se ha transformado en un desafío para los médicos veterinarios.
Estudios han demostrado que son principalmente 3 los sistemas orgánicos causantes de malos rendimientos, siendo el principal de ellos el sistema musculo esquelético, seguido del sistema respiratorio en su porción proximal y en tercer lugar, el sistema cardiovascular.

Estudios han demostrado que la evaluación de equinos F.S. de Carrera con bajo rendimiento, aproximadamente un $22 \%$ de ellos se debe a una cardiopatía primaria o secundaria (Martin, et al, 1999). Por lo anterior, es importante incluir una adecuada evaluación del sistema cardiovascular en los ejemplares que presenten malos rendimientos.

La técnica electrocardiográfica supone un importante método diagnóstico en el campo de la cardiología veterinaria moderna. Una evaluación clínica completa del sistema cardiovascular, debe necesariamente incluir un estudio electrocardiográfico, ya que éste es el método más exacto del que se dispone para evaluar y caracterizar trastornos del ritmo y conducción eléctrica cardiaca (Fregin, 1985).

\section{SISTEMA DE CONDUCCIÓN CARDÍACA DEL EQUINO}

El sistema de conducción del equino, en general posee una actividad eléctrica ordenada y tiene, en condiciones normales, una cadencia rítmica, que permite la contracción asincrónica de los atrios y los ventrículos (Lightowler, 2006). Este sistema de conducción está compuesto por: Nódulo sinoatrial (SA) o de Keith y Flack, nódulo atrioventricular (AV) o de Aschoff-Tawara, haz de His, sus ramas derecha e izquierda y por la red de Purkinje. El nódulo SA es el marcapasos normal del corazón y se ubica en la aurícula derecha, justo en la unión de ésta con la vena cava craneal (Colahan et al, 1998).

En el equino, el nódulo SA tiene la particularidad de ser una estructura relativamente grande con forma de herradura, localizándose en forma superficial, inmediatamente por debajo del epicardio. Esta característica anatómica, hace que la actividad eléctrica se expanda en forma radial a través del atrio derecho, generando el primer componente de la onda P bimodal en el ECG (Physick-Sheard, 1998; Doherty y Valverde, 2006). La depolarización de la aurícula izquierda parece ocurrir en forma espontánea sin contri- 
buir con la formación de la onda P del ECG en la superficie corporal, siendo la depolarización del tabique interatrial el segundo componente de la onda P bimodal (Physick-Sheard, 1998; Doherty y Valverde, 2006).

La onda $\mathrm{P}$ representa el auriculograma del ECG; es típicamente positiva aunque no es raro encontrarla bifásica (componente positivo y componente negativo) (Lightowler, 2006).

La conducción desde el nódulo SA hacia el AV, aún no ha sido confirmada y al parecer ocurre tanto por transmisión a través de células miocárdicas como por el tejido especializado de conducción. El retardo en la conducción producido a través del nódulo $\mathrm{AV}$, es el responsable del intervalo $\mathrm{PQ}(\mathrm{R})$ (Colahan et al, 1998). Por lo tanto, el tiempo de conducción atrioventricular $\mathrm{PQ}(\mathrm{R})$, corresponde al tiempo que demora la activación ventricular luego de haberse iniciado el marcapaso auricular, por ende, abarca el tiempo requerido para la depolarización auricular, el retardo que sufre el impulso en el nódulo atrioventricular y el tiempo de conducción a través del haz de His y sus ramas, hasta el comienzo de la depolarización ventricular (Godoy, 1988).

El haz de His es la única vía de conducción a través del tabique fibroso atrioventricular. La rama atrioventricular se divide en rama derecha e izquierda, que se dirigen hacia los ventrículos respectivos. Esta conducción en las ramas es más veloz que en el nódulo AV y la actividad pasa rápidamente hacia el miocardio ventricular a través de la red terminal de Purkinje.

El equino tiene la particularidad de tener una extensa arborización en la red terminal a través de todo el miocardio, desde el endocardio hasta el epicardio a diferencia de las otras especies (Colahan et al, 1998).

Para los no ungulados, como el caso de los humanos y otras especies, entre ellas el perro y el gato, cuando el impulso eléctrico llega a los ventrículos, lo primero en despolarizarse es la superficie endocárdica izquierda del tabique en su parte media, la que rápidamente compromete las dos superficies endocárdicas del tabique, generando un primer vector. Luego la depolarización se propaga por la superficie endocárdica de la pared libre de los dos ventrículos, generando un segundo vector, para finalmente extenderse hacia la parte externa y basal del corazón (parte superior de las paredes libres de ambos ventrículos y el septum interventricular), generando un tercer vector. En esta etapa de depolarización, el potencial eléctrico instantáneo se dirige hacia el ápice del corazón. (Lightowler, 2006).

En los ungulados, la depolarización ocurre de una manera distinta y lo primero en depolarizarse es la parte apical del septum interventricular de izquierda a derecha, generando un vector semejante al que se produce en los mamíferos no ungulados, y correspondería a la onda $\mathrm{Q}$ del ECG (Patteson, 1996; Physick-Sheard, 1998).

Como consecuencia de la gran arborización y diseminada red de Purkinje, la despolarización continúa en forma explosiva a partir de múltiples focos a través de la pared libre de ambos ventrículos, de tal manera que la mayor actividad eléctrica se cancela entre sí, por lo que no se genera deflexión en el electrocardiograma de superficie (Godoy, 1988; Patteson, 1996; Physick-Sheard, 1998). La actividad eléctrica que se registra en el ECG, es debida a la depolarización de una pequeña porción del ventrículo izquierdo que no alcanza a ser cancelada entre sí, generando un pequeño vector, correspondiendo a la onda R del ECG (Physick-Sheard, 1998; Lightowler, 2006). Finalmente, se produce un tercer vector, el que prácticamente origina todo el potencial que se inscribe como complejo ventricular y corresponde a la depolarización de la porción basal del tabique interventricular y de la pared libre ventricular, originando la onda S del ECG (Godoy, 1988; Patteson, 1996; Physick-Sheard, 1998). En esta etapa de despolarización, el potencial eléctrico instantáneo se dirige hacia la base del corazón a diferencia de otras especies (Patteson, 1996).

La repolarización ventricular ocurre primero en las superficies externas del corazón y especialmente la que se encuentra próxima a la punta del corazón. Luego ocurre en las superficies endocárdicas. Estas características fisiológicas dan el trazado característico de la onda T del ECG.

\section{MÉTODO Y TÉCNICA DE OBTENCIÓN DE UN ELECTROCARDIOGRAMA EN EL EQUINO}

Hoy en día existe una gran diversidad en lo que se refiere a la colocación de electrodos en la especie equina, como por ejemplo: sistema semiortogonal, sistema tetraédrico, sistema de derivación bipolar simple, sistema de "Dubois" 
y sistema de "Blanchard" (Colahan et al, 1998). Sin embargo, el sistema más utilizado desde hace muchos años, es el sistema clásico de Einthoven. El sistema de Einthoven se basa en la exploración del corazón mediante las derivaciones clásicas unipolares (aVR, aVL y aVF) y bipolares (D I, D II y D III), además del estudio de las derivaciones precordiales (CVCH, CV6RL, CV7LL y V10). (Godoy 1988).

La colocación de electrodos en otras partes del cuerpo del animal, hace que algunas conclusiones obtenidas con una técnica no puedan ser extrapoladas a otra técnica diferente (Lightowler, 2006).

En la búsqueda de un método de registro rápido y práctico para el sistema clásico de los miembros, se han intentado diferentes tipos y ubicaciones de los electrodos (Patteson, 1996). De esta manera, se han utilizado electrodos metálicos no polarizables de resistencia baja y estable, ubicados previa depilación en el tercio medio de la cara dorsal de los metacarpos y metatarsos derecho e izquierdo. Este sistema produce rechazo por los dueños debido a razones estéticas y por otro lado, los trazados electrocardiográficos no son de buena calidad (Dörner, 2009).

También se han usado electrodos de placa por caimanes ubicados directamente sobre la piel del animal previamente depilada y empastada con gel conductor ubicados en la parte caudal de los miembros anteriores, levemente por encima del olecranon y en los miembros posteriores, distal y lateral a la articulación femorotibiorrotuliana (Fregin, 1985).

Hoy en día se prefiere como electrodos

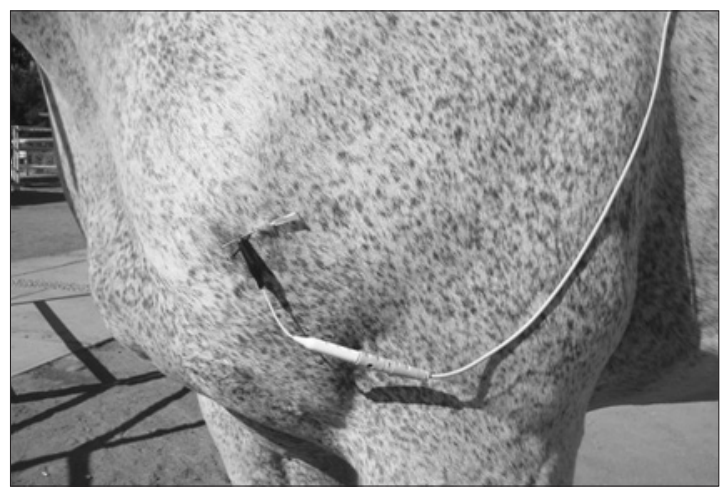

Figura 1. Electrodo explorador ubicado en el ángulo escápulo humeral izquierdo. agujas hipodérmicas de 21 G 11/2" las cuales han demostrado experimentalmente tener una buena tolerancia, buena conducción y una disminución de la resistencia eléctrica de la piel, sin causar ningún deterioro estético en el animal, ubicándolos subcutáneamente a nivel de la línea del encuentro (ángulos escápulo humerales y fosas del ijar) para las derivaciones estándar y unipolares de los miembros (Godoy, 1988). (Figura 1).

Por otro lado, se ha utilizado para las derivaciones precordiales, un electrodo en el sexto espacio intercostal izquierdo (CV7LL), otro electrodo ubicado en el sexto espacio intercostal derecho (CV6RL), ambos a la altura de la línea del encuentro (línea imaginaria trazada paralela al piso desde la articulación escápulo humeral). También se ubica un electrodo a nivel del músculo pectoral superficial, en la línea media entre las dos articulaciones escápulo humerales $(\mathrm{CVCH})$, y por último para completar el estudio eléctrico del corazón, se coloca un electrodo a la altura de la cruz (V10) (Fregin, 1985; Dörner, 2009).

Es importante señalar que la técnica de registro en el equino, como la forma del electrocardiograma, se ve significativamente influenciada por variaciones en la actividad del sistema nervioso autónomo (excitación y/o ejercicio), como también por cambios en la posición de los miembros anteriores (Fregin, 1982 a; Fregin, 1985).

Estas variaciones se pueden obviar, practicando el registro electrocardiográfico en la pesebrera habitual del equino, sujeto de su cuidador (Fregin, 1982 a, 1982 b). El piso debe estar seco y alejado de instrumentos o fuentes eléctricas que pudiesen hacer interferencia con el registro. Se debe respetar con exactitud la ubicación de las agujas y electrodos en los sitios pre-establecidos (Dörner, 2009). En lo posible, no se debe utilizar ningún tipo de tranquilizante ni anestésico.

La velocidad del registro es de $25 \mathrm{~mm} / \mathrm{seg}$, de modo que cada milímetro lineal represente una duración de $0,04 \mathrm{seg}$, con una estandarización de $1 \mathrm{~cm}$ por milivolt $(1 \mathrm{~mm}=0,1 \mathrm{mV})$ (Rose y Hodgson, 1995; Physick-Sheard, 1998). (Figura 2).

\section{REGISTRO ELECTROCARDIOGRÁFICO: ASPECTOS GENERALES}

Cuando ya se ha obtenido el electrocardiogra- 


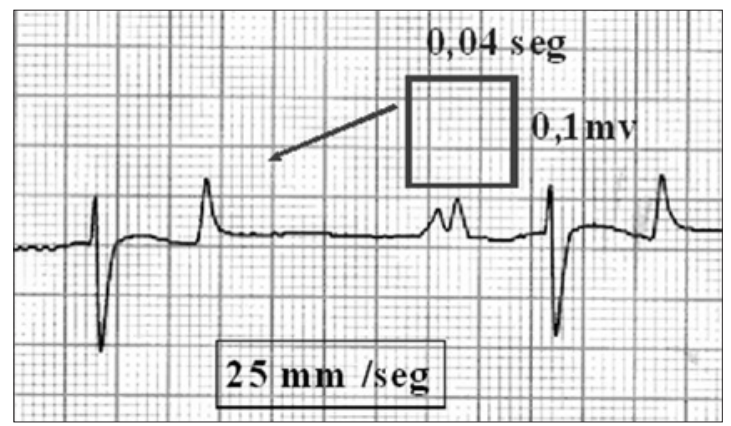

Figura 2. La velocidad del registro de $25 \mathrm{~mm} / \mathrm{seg}$., hace que cada milímetro lineal represente una duración de $0,04 \mathrm{seg}$, con una estandarización de $1 \mathrm{~cm}$ por milivolt $(1 \mathrm{~mm}=0,1 \mathrm{mV})$.

ma, se debe realizar una evaluación exhaustiva y sistemática del trazado (Fregin, 1985). Generalmente, la derivación de elección para este procedimiento, es la segunda derivación estándar (D II), con la cual podemos establecer el ritmo y frecuencia cardiaca y analizar las diferentes deflexiones, (Figura 3). En algunos casos se requiere el análisis del electrocardiograma completo con estos fines (Fregin, 1982 a).

El fenómeno de activación auricular (onda P) ha sido considerablemente estudiado en el equino F.S. de Carrera en lo que se refiere a su duración, amplitud y configuración. Lightowler (2006) señala una duración de la onda P de 0,14 $\pm 0,03$ segundos y una amplitud de $0,30 \pm 0,09$ milivolts.

El retardo en la conducción producido a través del nódulo $\mathrm{AV}$, es el responsable del intervalo $\mathrm{PQ}(\mathrm{R})$ (Colahan et al, 1998). Se ha descrito que para la medición del intervalo $P Q(R)$, es adecuado utilizar la derivación bipolar D II, aunque es más seguro utilizar la derivación en la cual halla una onda $\mathrm{P}$ bien formada y ancha, $\mathrm{y}$ una onda Q profunda (Godoy, 1988). El intervalo $\mathrm{PQ}(\mathrm{R})$ se mide desde el inicio de la onda $\mathrm{P}$ hasta el comienzo del complejo QRS (Rose y Hodgson, 1995), mientras que el segmento $P-Q(R)$ se considera como un período isoeléctrico, en el cual no se registran diferencias de potencial y se mide desde el final de la onda P hasta el comienzo del complejo QRS (Lightowler, 2006). Se describen valores de 0,3248 $\pm 0,037$ en el equino FSC.

La onda $\mathrm{T}$ es una onda alta, empinada e imprecisa pero de menor amplitud y más ancha que el complejo QRS, sin embargo, no es raro que sea más alta que el complejo QRS en la misma

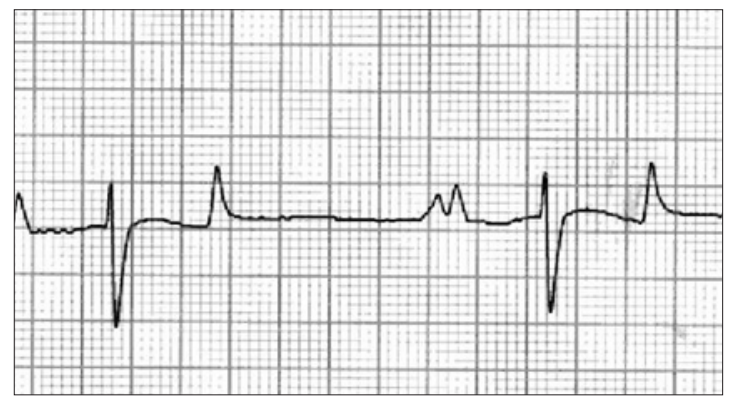

Figura 3. Registro electrocardiografico, representando la derivación bipolar II.

derivación. Se ha considerado como una onda T anormal cuando se presenta con mayor amplitud de lo normal y picuda o cuando se presenta con polaridad invertida (Muñoz et al, 2005). El segmento ST indica un período de reposo eléctrico, que media entre la activación ventricular y la repolarización ventricular; se mide desde el final del complejo QRS hasta el comienzo de la onda $\mathrm{T}$ (Doherty y Valverde, 2006). En general es isoeléctrico, pero se consideran normales desviaciones de $\pm 0,1$ milivolts (Lightowler, 2006).

El intervalo QT señala la duración del sístole ventricular, es decir representa el tiempo transcurrido para la despolarización y repolarización de la musculatura ventricular y coincide estrechamente con el sístole mecánico ventricular, puesto que éste comienza exactamente con el vértice de la onda $\mathrm{R}$ y termina con el fin de la onda $\mathrm{T}$ (Godoy, 1988; Rose y Hodgson, 1995). Se mide desde el comienzo del complejo QRS hasta el fin de la onda $\mathrm{T}$ en la derivación en la cual aparece más largo.

\section{REGISTRO ELECTROCARDIOGRÁFICO: VARIACIONES}

La onda P ha sido muy estudiada y se describe que el primer componente de esta onda es el más lábil, pudiendo sufrir pequeños cambios hasta una completa inversión, siendo el segundo componente menos afectado por el sistema nervioso autónomo (Fregin, 1982 b). En una misma derivación, los cambios de la onda P pueden persistir durante varios latidos antes de recuperar su forma original, este fenómeno se denomina mi- 
gración del marcapaso (Rose y Hodgson, 1995; Godoy, 2008). Los cambios de la configuración de la onda $\mathrm{P}$ pueden deberse a variaciones de la actividad del sistema nervioso autónomo o a un proceso de dilatación auricular (Fregin, 1982 a; Fregin, 1985; Godoy, 2008).

En condiciones normales, el equino tiene una fuerte influencia del sistema neurovegetativo, y por ello, existen prolongaciones vagotónicas que llevan al desarrollo de bloqueos atrioventriculares de primero y segundo grado completamente funcionales (Lightowler, 2006). Además se describe una correlación positiva de la edad con la duración del intervalo $\mathrm{PQ}(\mathrm{R})$ y negativa con la frecuencia cardíaca (Lightowler, 2006).

La onda $\mathrm{T}$ es una de las deflexiones más variables del ECG (Stewart et al, 2006; Rose y Hodgson, 1995; Muñoz et al, 2005), y puede ser influenciada por muchos factores fisiológicos y/o patológicos, como el ejercicio, excitación, drogas, enfermedades infecciosas, tono vagal, desbalance electrolítico, hipoxia y miocarditis (Stewart et al, 1983; Muñoz et al, 2005). Como la onda $\mathrm{T}$ puede ser influenciada por una gran número de factores, cuantificar la significancia de su diagnóstico es difícil (Muñoz et al, 2005).

Las anormalidades de la onda $\mathrm{T}$ se han relacionado con disminución del tiempo de llenado ventricular, volumen minuto reducido, gasto cardiaco y rendimiento en el ejercicio en caballos de carrera (Stewart et al, 1983; Muñoz et $a l, 2005)$. Se ha reportado una disminución de la capacidad aeróbica en caballos con alteraciones de la repolarización ventricular, lo que sugiere una disminución de la contractibilidad miocárdica en algunos casos (Stewart et al, 1983). En general la onda $\mathrm{T}$ no presenta melladuras ni bifideces (Godoy, 1988).

La evaluación de la duración del intervalo ST no constituye un parámetro importante en la electrocardiografía clínica (Dörner, 2009), sin embargo, las desviaciones por sobre o bajo la línea isoeléctrica mayor a 0,1 milivolt, es indicativo de falla cardiaca, siempre y cuando se descarte una alteración neurógena del fenómeno (Godoy, 1988). El segmento ST resulta difícil de medir en algunas ocasiones (Doherty y Valverde, 2006). También se registran valores para el intervalo ST de 0,259 $\pm 0,011$ segundos en D II.

La duración del intervalo QT es altamente dependiente de la frecuencia cardíaca (Rose y Hodgson, 1995; Lightowler, 2006), acortándose al aumentar la frecuencia cardiaca y alargándose al aumentar ésta, por lo que el valor observado debe expresarse corregido como si el paciente hubiese mostrado una frecuencia cardiaca uniforme propia de la especie en condiciones normales. Para ello se utiliza la fórmula de Bazzet (Lightowler, 2006).

$$
\begin{array}{r}
\text { QTc }=\text { QT observado } \\
\text { Intervalo R-R }
\end{array}
$$

Hoy en día, sin lugar a dudas, un tema importante que provoca cierta inquietud en la hípica, se refiere a dilucidar clínicamente las causas que originan malos rendimientos. Después de los desordenes musculo esqueléticos y de vías respiratorias altas, las anormalidades cardiacas son la tercera causa de disminución del rendimiento en caballos Fina Sangre de Carrera (Martin et al, 1999).

Muchos de los ejemplares que presentan bajos rendimientos, presentan un ECG alterado y se ha detectado una gran incidencia de anormalidades de la onda $\mathrm{T}$ en ejemplares con bajos rendimientos (Stewart et al, 2006).

Las arritmias se pueden clasificar como fisiológicas y patológicas, dentro de las primeras, las más frecuentemente encontradas son el bloqueo atrio ventricular de primer grado y de segundo grado mobitz tipo 1 (Stewart et al, 2006), bradicardia sinusal y arritmia sinusal (Van Loon, 2005). Estas arritmias fisiológicas son de carácter benigno funcional y se presentan en el momento de reposo del animal debido a un aumento del tono vagal (Van Loon, 2005; Godoy, 2008). Es importante mencionar que las arritmias fisiológicas también pueden ocurrir inmediatamente posterior al ejercicio debido a un cambio del tono del sistema nervioso autónomo (Van Loon, 2005).

Por otro lado, las arritmias patológicas más importantes son fibrilación atrial (Van Loon, 2005; Stewart et al, 2006), latido prematuro atrial y taquicardia atrial, latido prematuro ventricular y taquicardia ventricular, bloqueo atrio ventricular de segundo grado mobitz tipo 2 y bloqueo atrio ventricular de tercer grado (Van Loon, 2005).

Las arritmias atriales se caracterizan electrocardiográficamente por alteraciones de la onda $\mathrm{P}$, lo que se da generalmente con un complejo QRS y onda T normales (Godoy, 1988). 
La taquicardia ventricular se caracteriza por un registro con una alta frecuencia cardiaca y con acortamiento del intervalo R-R (Fregin, 1982 b).

El bloqueo atrio ventricular de primer grado se caracteriza por un aumento del intervalo P-Q(R) a más de 0,44 segundos, manteniéndose el ritmo sinusal, mientras que en el bloqueo de segundo grado se produce un registro en el cual aparecen dos o más ondas $\mathrm{P}$ que preceden al complejo QRS (Godoy, 2008).

Las arritmias ventriculares en el equino son menos frecuentes, pero más indicativas de patologías, siendo el complejo QRS y la onda T los principales parámetros electrocardiográficos alterados (Godoy, 1988).

El examen electrocardiográfico equino se puede utilizar para otras aplicaciones clínicas como por ejemplo, caballos con epistaxis, hemorragia pulmonar oculta o individuos con síndrome cólico (Godoy, 1988).

Enequinosfinasangre decarrera"sangradores" se ha determinado que las principales alteraciones electrocardiográficas se observan a nivel del fenómeno de activación auricular, presentándose la onda $\mathrm{P}$, predominantemente bimodal, a veces trimodal, de mayor duración, con desviación del eje promedio de activación auricular y con un alto porcentaje de migración del marcapasos (Godoy, 2008).

Las alteraciones que se presentan predominantemente en el síndrome cólico, son los latidos ventriculares prematuros. Se describe una conexión entre la disfunción cardiaca y una estimulación anormal del sistema nervioso primariamente, además de un desbalance hemodinámico y una disminución del metabolismo del oxigeno como factores secundarios (Reed et al., 2004) Además se describe asociado a la presentación de latidos prematuros ventriculares, la aparición de bloqueos atrio ventriculares de primer y segundo grado en forma secundaria. En estos casos un tratamiento especial no es necesario debido a que las irregularidades cardíacas desaparecen a medida que los síntomas del síndrome cólico desaparecen (Reed et al, 2004).

\section{VECTOCARDIOGRAFÍA. ANÁLISIS VECTORIAL DEL CORAZÓN}

El corazón tiene un eje anatómico y otro eléctrico, los cuales no son idénticos. Nos interesa analizar el eje eléctrico, el cual se representa por una flecha o vector con dirección, magnitud y sentido, sobre el plano horizontal (frontal). Dicho vector es el resultante de las innumerables fuerzas eléctricas que se originan en el músculo cardiaco durante la activación ventricular y auricular, y la repolarización ventricular, las cuales se estudian a través del cálculo de los ejes promedios de activación auricular, ventricular y de repolarización ventricular (Godoy, 1988; Colahan et al, 1998). Por lo tanto, el estudio electrocardiográfico del corazón, se completa al conocer las características vectoriales del fenómeno eléctrico cardiaco.

El análisis vectorial del electrocardiograma ha sido ampliamente estudiado tanto para el humano como para el equino. En el humano, el vector principal de la depolarización ventricular se dirige hacia el ápice del corazón, o sea, hacia la masa ventricular predominante. (Lightowler, 2006). El equino a diferencia del hombre, tiene un eje eléctrico medio ventricular con dirección hacia la base cardíaca y no hacia la masa ventricular predominante (Patteson, 1996; Colahan et al, 1998).

Para la determinación de los ejes de activación auricular y ventricular, como el eje de repolarización ventricular, la información recogida de dos derivaciones bipolares y dos derivaciones unipolares, son llevadas al sistema hexaxial de Bayley (Godoy, 1988; Colahan et al., 1998). Para ello se realiza la suma algebraica de la amplitud entre dos derivaciones bipolares y entre dos derivaciones unipolares ya sea para $\mathrm{P}, \mathrm{QRS}$ o $\mathrm{T}$, y los valores obtenidos se trasportan al sistema hexaxial de Bailey. Luego se trazan líneas perpendiculares a las 2 derivaciones elegidas y se calcula el vector resultante el cual es el vector medio ya sea de P, QRS o T (Colahan et al., 1998).

El sistema hexaxial de Bailey se basa en que los tres lados del triángulo de Einthoven son desplazados hacia el centro, lugar donde teóricamente se encuentra el corazón. Se obtiene un sistema de seis ejes con las derivaciones unipolares y bipolares, en el plano frontal para el humano $\mathrm{u}$ horizontales para el equino. Las tres derivaciones bipolares en este sistema constan de una parte positiva y otra negativa: la parte positiva de D I se sitúa a $0^{\circ}$ y la negativa a \pm $180^{\circ}$; la parte positiva de D II a $+60^{\circ}$ y la negativa a $-120^{\circ}$; la parte positiva de D III se sitúa a $+120^{\circ}$ 
y la negativa a $-60^{\circ}$; la parte positiva de aVR está a $-150^{\circ}$ y la negativa a +30 , la parte positiva de aVL está a $-30^{\circ}$ y la negativa a $+150^{\circ}$, y la parte positiva de $\mathrm{aVF}$ a $+90^{\circ}$ y la negativa a $-90^{\circ}$.

\section{CONCLUSIONES}

En la actualidad, existe una seria preocupación por dilucidar la causa de los malos rendimientos en los equinos de deporte y en la clínica veterinaria moderna ha cobrado mucha importancia el examen cardiovascular, el cual debe incluir invariablemente la obtención de un registro electrocardiográfico.

Es importante que el médico veterinario tenga el conocimiento de todos los cambios que puede sufrir un equino a nivel cardiaco en relación a la edad, género, condición de entrenamiento o en relación a alguna alteración patológica concomitante para poder realizar una correcta interpretación de la anormalidad observada.

$\mathrm{Si}$ bien es cierto, la vectocardiografía ha sido estudiada en el equino por largos años, no existen resultados concretos estadísticamente significativos que nos indique alguna utilidad clínica, situación que es aún discutida.

El estudio completo del sistema cardiovascular en el equino fina sangre de carrera debiera incluir además de la evaluación electrocardiografía, un completo estudio eco cardiográfico y un test de esfuerzo junto a la medición de los parámetros sanguíneos hematológicos y bioquímicos.

\section{REFERENCIAS}

1.- COLAHAN P, MAYHEW I, MERRITT A, MOORE J. 1998. Medicina y Cirugía Equina: Vol. I. Cuarta Edición. Editorial Intermédica. Buenos Aires. Argentina. $1736 \mathrm{p}$.

2.- DOHERTY T, VALVERDE A. 2006. Manual of Equine Anesthesia and Analgesia. Primera Edición, Editorial Blackwell Publishing. UK. 362 p.

3.- DÖRNER C. 2009. Evaluación Electrocardiográfica de Equinos Fina Sangre de Carrera Clínicamente Sanos en Período de Amansa. Tesis. Santiago, Chile. U. Chile, Fac. Cs. Veterinarias y Pecuarias. 106 p.
4.- FREGIN GF. 1982 a. The Equine Electrocardiogram with Standardized Body and Limb Positions. Cornell, Vet 72 (3): 304-324.

5.- FREGIN GF. 1982 b. The Cardiovascular System. Equine Medicine and Surgery. Santa Bárbara, California. American Veterinary Publications, Inc. pp. 645 - 704 (citado por Godoy, A. 1988. In:_Caracterización Electrocardiográfica de Equinos Pura Sangre Chilenos Clínicamente Sanos. Tesis. Santiago, Chile. U. Chile, Fac. Cs. Veterinarias y Pecuarias. 135 p.).

6.- FREGIN GF. 1985. Electrocardiography. Vet Clin of North Am 1: 419-435.

7.- GODOY A. 1988. Caracterización Electrocardiográfica de Equinos Pura Sangre Chilenos Clínicamente Sanos. Tesis. Santiago, Chile. U. Chile, Fac. Cs. Veterinarias y Pecuarias. $135 \mathrm{p}$.

8.- GODOY A. 2008. Cardiología Equina [grabación audio]. Santiago, Chile. U. Chile, Fac. Cs. Veterinarias y Pecuarias. Departamento de Ciencias Clínicas. 1 Cinta (Digital), 60 minutos. Sonido.

9.- LIGHTOWLER CH. 2006. Manual de Cardiología del Caballo. Primera Edición. Editorial Chinfield S.A. Argentina. $1736 \mathrm{p}$.

10.- MARTIN JR B, REEF V, PARENTE E. 1999. Clinical Evaluation of Poor Training or Racing Performance in 348 horses (1992-1996). AAEP PROCEEDINGS 45: 322-324.

11.- MUÑOZ A, SATUE K, ROVIRA S, LUCAS RG, BENITO M. 2005. Electrocardiographic Ventricular Repolarisation Processes in Andalusian Horses Before and After Physical Training. Bulgarian Journal of Veterinary Medicine 8 (1): 23-34.

12.- PATTESON M. 1996. Equine Cardiology. Primera Edición. Editorial Blackwell. London. [en línea]http:// www.provet.co.uk/equinecardiology/ [consulta: 19-032010].

13.- PHYSICK-SHEARD PW. 1998. Enfermedades del Aparato Cardiovascular. In: Medicina y Cirugía Equina. Vol. I. Cuarta Edición. Editorial Intermédica. Buenos Aires. Argentina. pp 151-162.

14.- REED S, BAYLY W, SELLON D. 2004. Equine Internal Medicine. Segunda Edición. Editorial Saunders. U.S.A. $1659 \mathrm{p}$.

15.- ROSE RJ, HODGSON DR. 1995. Manual Clínico de Equinos. Primera edición. Editorial Mcgraw Hill Interamericana. México. $632 \mathrm{p}$.

16.- STEWART JH, ROSE RJ, DAVIS PE, HOFFMAN K. 2006. A Comparison of Electrocardiographic Findings in Racehorses Presented either for Routine Examination or Poor Racing Performance. Equine Exercise Physiology. Snow, D.H., S.G.B. Persson a R.J. Rose (Eds.), Cambridge Granta Editions.

17.- VAN LOON G. 2005. Reduced Performance Due to a Bad Engine?. Department of Large Animal Internal Medicine, Ghent University, Belgium. 